\title{
Valoración de la inserción de la inteligencia artificial en el ámbito jurídico: un análisis frente al proceso del ente acusador en etapa de indagación*
}

\author{
[Artículos]
}

\author{
María Fernanda Castro Colmenares ${ }^{* *}$ \\ Nicolás Eduardo Sánchez Cortez ${ }^{* * *}$ \\ Lina María Ortegón Suárez ${ }^{* * * *}$
}

Fecha de recepción: 4 de marzo de 2021

Fecha de aprobación: 18 de mayo de 2021

\footnotetext{
* Este artículo es producto del proyecto Migración y Trata de Personas (COL0201684) y del proyecto La Inteligencia Artificial y el Derecho (IC2020-FSCC-DSIC-31) que forma parte del grupo de investigación Derecho, Sociedad y Empresa, línea de investigación de escuela "Derecho constitucional, procesal y de MASC" de la Institución Universitaria Politécnico Grancolombiano (Bogotá, Colombia).

** Estudiante de Derecho de la Institución Universitaria Politécnico Grancolombiano (Bogotá, Colombia). Miembro del referido semillero. Correo electrónico: macastro27@poligran.edu.co. ORCID: https://orcid.org/0000-0003-1039-4632

*** Estudiante de Derecho de la Institución Universitaria Politécnico Grancolombiano (Bogotá, Colombia). Miembro del referido semillero. Correo electrónico: nisanchez12@poligran.edu.co. ORCID: https://orcid.org/0000-0002-6543-3961

**** Abogada, especialista en Derecho Administrativo y magíster en Derecho Procesal de la Universidad Libre (Colombia). Coordinadora Académica del área Derecho Procesal y Calidad, docente de pregrado de la Institución Universitaria Politécnico Grancolombiano. Tutora y directora del referido semillero Estudios en Derecho Procesal con énfasis en la eficacia del bloque de constitucionalidad. Correo electrónico: Imortegon@poligran.edu.co. ORCID:

https://orcid.org/0000-0001-9971-5070
}

Via Inveniendi Et Iudicandi

e-ISSN: 1909-0528 | DOI: https://doi.org/10.15332/19090528

Vol. 16 N.0 2 | julio-diciembre del 2021 
Citar como

Castro Colmenares, M. F., Sánchez Cortez, N. E. y Ortegón Suárez, L. M. (2021).

Valoración de la inserción de la inteligencia artificial en el ámbito jurídico: un análisis frente al proceso del ente acusador en etapa de indagación. Via

Inveniendi Et Iudicandi, 16(2). https://doi.org/10.15332/19090528.6787

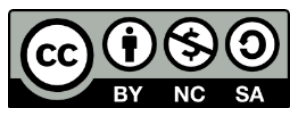

\section{Resumen}

El presente artículo aborda la relación que puede existir entre la inteligencia artificial (IA) y el sistema penal acusatorio, específicamente como coadyuvante para la Fiscalía, pretendiendo que, por medio de los hechos y el material probatorio recogido por parte de la policía judicial, este sea analizado por un software, el cual mediante la información obtenida logre determinar la conducta delictiva. Esto por medio de una investigación cualitativa con aplicación de un método teórico deductivo, que consiste en la revisión de bases de datos, bibliografía y estadística, iniciando con la exposición de la función de la fiscalía en fase de indagación, continuando con un análisis estadístico, la informática jurídica y la ubicación de la inteligencia artificial entre sus ramas, un estudio de derecho comparado con México, Argentina, Estados Unidos, Canadá y Australia, para dar respuesta al problema jurídico identificado frente a la inadecuada tipificación de las conductas punibles, lo cual a su vez vulnera las diferentes garantías procesales. Lo anterior, mediante la generación de recomendaciones para la implementación de un software que gestione y analice las circunstancias fácticas y jurídicas para determinar con mayor facilidad la conducta.

Palabras clave: inteligencia artificial, informática jurídica, sistema penal acusatorio, fiscalía, fase de indagación. 


\section{Assessment of the insertion of artificial intelligence in the legal field: an analysis of the prosecuting entity in the investigation stage}

\section{Abstract}

This article addresses the relationship that may exist between artificial intelligence (AI) and the accusatory criminal system, specifically as an assistant for the Prosecutor's Office, claiming that, through the facts and the probative material collected by the judicial police, the latter is analyzed by a software, which can determine the criminal conduct through the information obtained. This through a qualitative research with the application of a theoretical deductive method, consisting of the review of databases, bibliography and statistics, beginning with the presentation of the function of the prosecutor's office in the investigation phase. It continues with a statistical analysis, legal informatics and the location of artificial intelligence among its branches, a study of comparative law with Mexico, Argentina, United States, Canada, and Australia, to respond to the legal problem identified in the face of the inadequate classification of punishable conducts, which in turn violates the different procedural guarantees. The above, by generating recommendations for the implementation of a software that manages and analyzes the factual and legal circumstances to determine more easily the conduct.

Keywords: artificial intelligence, legal informatics, accusatory criminal system, prosecution, investigation phase.

\section{Introducción}

A lo largo de la historia, las actividades ejercidas por el ser humano se han visto influenciadas por los avances tecnológicos, creando a su vez la necesidad de adaptación a las diferentes herramientas informáticas. Entre los avances más importantes de los últimos sesenta años se encuentra la 
llamada inteligencia artificial (IA), cuya primera definición fue dada en 1956, esta responde a la creación de máquinas o artefactos capaces de ejecutar actividades de manera inteligente, tal y como un ser humano podría hacerlo.

Por medio del estudio de sistemas informáticos e investigación científica, la IA ha procurado dar solución a problemas complejos, desarrollando, por medio de la tecnología, mecanismos capaces de resolver satisfactoriamente diferentes tareas en variedad de entornos. Para coadyuvar en dicho estudio de automatización, se abordan temas matemáticos, biológicos, lingüísticos, psicológicos, físicos, entre otros.

Entre la variedad de temas comprendidos se encuentra también el campo jurídico, en el cual la implementación de sistemas informáticos ha tenido alto impacto, pues por medio de diferentes programas y aplicaciones se ha logrado la optimización del aparato judicial. Esto se ha dado especialmente en Países Bajos, donde autoridades en derecho como tribunales y juzgados, han decidido acoger herramientas dirigidas a una gestión automática.

Es vital tener claros a qué se refieren ciertos conceptos como informática jurídica, tecnología de la información e inteligencia artificial en derecho. La informática jurídica se puede definir como aquella técnica propia del derecho para la recuperación de información jurídica, la cual se recopila con el fin de ser utilizada para el desarrollo y análisis de conceptos jurídicos (Saavedra, Saavedra y Montaña, 2018). Otra definición de la informática jurídica es la aplicación de instrumentos tecnológicos a las diferentes operaciones que se realizan en el ámbito del derecho, es decir que es una herramienta utilizada por quienes ejercen el derecho (Peña, 2014). 
La informática jurídica ha sido clasificada en tres conceptos, informática jurídica de gestión, informática jurídica documental e informática jurídica decisoria, la primera se entiende como aquella que los abogados, jueces y peritos ejercen para realizar tareas cotidianas como almacenamiento de datos, escritura y comunicación, la segunda es con la cual se busca recuperar documentos jurídicos, es decir leyes, sentencias y doctrina haciendo más fácil la tarea de buscar jurisprudencia por medio de cualquier palabra que haga referencia a determinado tema que se pretende buscar, y la tercera es aquella que va ligada con la inteligencia artificial, ya que por medio de esta se busca crear sistemas o técnicas que simulen el razonamiento jurídico, por tanto, es la rama de mayor interés en esta investigación (Peña, 2014).

La inteligencia artificial se define como aquellos sistemas que manifiestan un comportamiento inteligente y se comenzó a hacer visible en aparatos de reconocimiento facial o de voz es decir aquellos programas con objetivos específicos, pero que tenían cierta autonomía, actualmente podemos ver la inteligencia artificial en automóviles, robots o drones, por medio de algoritmos, redes neuronales artificiales y patrones de razonamiento. Hoy se pueden crear dispositivos que tienen la capacidad de razonar, tomar decisiones y cumplir a cabalidad con las tareas programados, sin intervención humana (Cotino, 2019).

El presente artículo decide traer a colación las funciones de la Fiscalía General de la Nación (FGN), específicamente señaladas en el art. 250 y subsiguientes en la Carta Suprema y es por ello por lo que surge la siguiente pregunta de investigación: ¿desde qué campo de la informática jurídica se articula la IA como una herramienta que facilite la imputación de cargos en el sistema penal? El presente artículo propone generar recomendaciones para la implementación de un software combinado con 
informática jurídica decisoria, propia del área de la IA, en la FGN, planteando que a través de un programa tecnológico se logre la debida imputación, analizando las circunstancias fácticas y jurídicas para tipificar con mayor facilidad la conducta por medio de la carga de datos existentes, y bajo la supervisión y auditoria de los funcionarios públicos expertos en el tema.

\section{Método}

Lo anterior, mediante una investigación teórica encaminada a un enfoque cualitativo que se adhiere a la conjetura que pretendemos desarrollar en el presente trabajo, al mostrar la pertinencia de aplicar, de forma sistematizada, la IA para generar eficacia a la hora de tipificar el delito en la formulación de imputación por parte de la FGN. La investigación trae consigo la inserción en el campo objeto de estudio con el fin de identificar situaciones que aporten datos encaminando al fiscal como director de la investigación en el desarrollo de esta; procesando información bajo un pensamiento lógico-racional, empleando herramientas tales como, consulta de bases de datos, artículos, trabajos de grado, libros, revistas y la legislación vigente, así como la consulta de datos reportados por la FGN en los últimos dos años. La presente investigación esboza un estudio sistemático, panorama propicio para la construcción de proposiciones, metodología propia de los estudios jurídicos en procedimiento (Villabella, 2015).

\section{Resultados}

\section{Funciones Fiscalía-Sistema Penal Acusatorio}

La FGN surgió bajo la promulgación de la Constitución Política de Colombia (C.P.) (1991), quedando con la dirección sobre la policía judicial, 
la cual tiene como función apoyar en la investigación penal (FGN, s. f.). Es decir que la FGN es la encargada de coordinar y dirigir funciones de policía judicial. De conformidad con lo establecido en el artículo 250 de la C.P., la FGN es la responsable de la acción penal desde la primera fase (indagación), la cual inicia desde la noticia criminal, por medio de la policía judicial, que se encarga de realizar la respectiva investigación, y contribuye en la formulación de acusación en cuanto haya recopilado los medios materiales probatorios y la información legalmente obtenida con el fin de ser introducida luego en el juicio.

Entre las características más relevantes del Sistema Penal Acusatorio, se encuentra la clasificación de autoridad competente, las etapas y las audiencias que cada una contempla, en un primer momento, dichos funcionarios son la policía judicial, la FGN y los jueces de control de garantías, que desarrollan la etapa de indagación que está contemplada desde las audiencias preliminares, hasta la audiencia de formulación de imputación.

En segundo lugar, se encuentran los jueces de conocimiento, quienes desarrollan las audiencias de formulación de acusación, preparatoria y de juicio oral, y, finalmente, en un tercer momento, encontramos los jueces de ejecución de penas, que, entre sus funciones, tienen la reparación de perjuicios materiales causados dentro de la acción penal y el cumplimiento de la sentencia (Sarmiento, Medina y Plazas, 2017). Es importante señalar que para el tema de interés del presente artículo se ahondará en la fase de indagación, propia de la actividad desarrollada en eventos única y exclusivamente por la policía judicial y en otras ocasiones en apoyo de la FGN.

La fase de indagación inicia cuando es interpuesta la noticia criminal o cuando se pone en conocimiento de las autoridades la ocurrencia de unos 
hechos que puedan inferir la posible comisión de un delito, a partir de ello, la policía judicial despliega una actividad investigativa, la cual, dependiendo el caso, requiere la autorización de la FGN (Gonzáles, 2007). En la etapa de investigación, el fiscal está en la facultad de ordenar todas las actividades, en tanto estas no vulneren los derechos fundamentales; algunas actuaciones, como los registros y allanamientos, requieren un control judicial posterior con el fin de ser legalizados, dicho control se debe hacer dentro de las 12, 24 o 36 horas siguientes, dependiendo del tipo de prueba en cuestión, por otro lado, la evidencia física y el material probatorios con el fin de preservar su autenticidad deben ser sometidos a cadena de custodia.

La actividad investigativa se lleva a cabo mediante los organismos de policía judicial (CTI, SIJÍN/DIJIN, Medicina Legal), quienes tienen como misión la recolección de material probatorio para que de dicha manera la FGN realice las peticiones pertinentes al juez (Rama Judicial. s. f.), esta etapa de indagación finaliza con la formulación de imputación, dónde se le informa a la persona el delito por el cual se le investiga y por cual continuará el proceso. En dicho momento, el fiscal puede también solicitar al juez la medida de aseguramiento que considere pertinente, por ello resulta tan importante la etapa de recolección de material probatorio. Existen ciertos requisitos en el desarrollo de la formulación de la imputación, por un lado, la correcta individualización, es decir, una información integral para poder facilitar la ubicación del sujeto e identificarlo plenamente con el fin de que exista la certeza que la misma persona que es señalada de cometer la conducta punible, es la que está compareciendo. Por otro lado, se encuentra la imputación jurídica y fáctica, la primera corresponde a la claridad que debe existir en la determinación del delito; por su parte, la imputación fáctica lleva inmersa 
la imputación subjetiva y objetiva, que en pocas palabras resultan siendo aquellos elementos intangibles que justifican la imputación (Hernández, 2009).

El sustento probatorio es otro requisito para la formulación de imputación, este resulta necesario para cumplir con el principio de legalidad que menciona la Constitución Política en el artículo 29 y, a su vez, para evitar una futura nulidad. Como último requisito, se encuentra la dosificación punitiva, que resulta ser la manifestación de la pena según la conducta que se desplegó (el delito).

Resulta ser entonces la formulación de imputación un momento procesal fundamental para dar a conocer la conducta que desplegó la acción penal y así mismo realizar la recolección previa de material útil para el proceso.

\section{Análisis estadístico y exponencial de los procesos penales acusatorios en la FGN del 2019 a 2020}

Colombia, a lo largo de su historia, ha enfrentado diversos conflictos sociales y políticos, dejando como consecuencia un alto índice de víctimas de la violencia y una tasa de homicidios de 77.5 por cada 100000 habitantes durante los años de 1987 hasta 1992 (Montenegro y Posada, 1994). El contexto actual es diferente, un ejemplo en cifras de esta realidad la podemos ver reflejada en el índice de homicidios registrado en 2017, el cual corresponde a 25 por cada 100 ooo habitantes (Tamayo y Norza, 2018, p. 49).

Teniendo esto en cuenta y el contexto actual, es pertinente empezar a hablar sobre los delitos existentes en Colombia, su regulación, los procesos y estadísticas. Como bien se mencionaba, la regulación y tipificación de los delitos está contemplada en la Ley 599 de 2000 o más conocido como Código Penal. En sus 476 artículos se puede encontrar un aproximado de

Via Inveniendi Et Iudicandi

e-ISSN: 1909-0528 | DOI: https://doi.org/10.15332/19090528

Vol. 16 N.0 2 | julio-diciembre del 2021 
1155 conductas punibles, las cuales son estudiadas por el fiscal a cargo del proceso para determinar la conducta delictiva que se desplegó producto de los hechos ejecutados por la persona o las personas señaladas en la denuncia, querella o petición especial que haya sido puesta en su conocimiento.

Cabe resaltar que, aunque varios delitos posean el mismo nombre, su tipificación es diferente, debido a las circunstancias de mayor o menor punibilidad, como atenuantes y agravantes en que incurra el sujeto que comete la conducta, ítems que son segregados por los datos públicos del ente acusador, como se muestra en la siguiente tabla, que se elaboró con base en los datos suministrados y discriminados por grupo de delito.

Tabla 1. Delitos presentados entre abril 2019 y julio 2020

\begin{tabular}{|c|c|c|}
\hline Grupo de delito & Suma de total procesos & Correspondencia en \% \\
\hline Delitos sexuales & 206501 & 3.037 \\
\hline Falsedad en documentos & 185688 & 2.731 \\
\hline Injuria y calumnia & 184186 & 2.709 \\
\hline Estafa & 184004 & 2.706 \\
\hline Fabricación, tráfico y porte de armas & 139870 & 2.057 \\
\hline Fe pública & 128875 & 1.895 \\
\hline Impartición de justicia & 108242 & 1.592 \\
\hline Delitos informáticos & 76174 & 1.120 \\
\hline Libertad individual y otras garantías & 71590 & 1.053 \\
\hline Administración pública & 66756 & 0.982 \\
\hline Corrupción administrativa & 65392 & 0.962 \\
\hline Seguridad pública & 55207 & 0.812 \\
\hline Constreñimiento & 49829 & 0.733 \\
\hline Corrupción judicial & 38581 & 0.729 \\
\hline Erden económico social & 30757 & 0.686 \\
\hline Desaparición forzada & 46657 & 0.561 \\
\hline Eomicidio culposo & 3859 \\
\hline
\end{tabular}

Via Inveniendi Et Iudicandi

e-ISSN: 1909-0528 | DOI: https://doi.org/10.15332/19090528

Vol. 16 N.0 2 | julio-diciembre del 2021 


\begin{tabular}{|c|r|r|}
\hline Grupo de delito & Suma de total procesos & Correspondencia en \% \\
\hline Corrupción tributaria & 27153 & 0.399 \\
\hline Recursos naturales y medio ambiente & 20958 & 0.308 \\
\hline Vida e integridad personal & 14150 & 0.208 \\
\hline Personas y bienes protegidos por el DIH & 11428 & 0.168 \\
\hline Secuestro simple & 10994 & 0.162 \\
\hline Régimen constitucional y legal & 8723 & 0.128 \\
\hline Derechos de autor & 8239 & 0.121 \\
\hline Salud pública & 6119 & 0.090 \\
\hline Corrupción electoral & 3857 & 0.057 \\
\hline Secuestro extorsivo & 3685 & 0.054 \\
\hline Participación democrática & 2670 & 0.039 \\
\hline Maltrato animal & 2565 & 0.038 \\
\hline Corrupción privada & 2434 & 0.036 \\
\hline Otros delitos & 1440 & 0.021 \\
\hline Seguridad del Estado & 51 & 0.001 \\
\hline Total & 6170 & 100 \\
\hline
\end{tabular}

Fuente: elaboración propia con base en datos de la FGN (2019-2020).

Como se puede observar en la tabla, las 1155 conductas punibles mencionadas fueron asociadas en un total de 41 grupos de delitos para lograr una mayor facilidad de análisis y comprensión. En términos generales, durante este periodo se registraron un total de 6799170 delitos a nivel nacional, siendo el hurto el delito más cometido, ya que corresponde a un poco más de la cuarta parte del total.

Estas cifras son bastantes preocupantes, puesto que los números registrados influyen en que se pueda dar un alto índice de criminalidad en el país. Además, se aumenta la carga laboral que cada fiscal tiene a su cargo, debido a que, en cada caso atendido por este, puede existir más de un delito cometido y, a su vez, cada uno de estos delitos deben ser identificados y especificados tal como se encuentra tipificado en el Código 
Penal para poder llegar a una justa determinación de pena por los actos cometidos.

Si se tiene en cuenta esta gran cantidad de carga laboral para los fiscales del país y la verificación de la efectividad de su trabajo, se pueden encontrar varios riesgos graves que se podrían presentar, entre estos están: no revisar detalladamente el caso, mala tipificación de la conducta delictiva, sesgo del fiscal, entre otros.

Tabla 2. Número de procesos en etapas fiscales

\begin{tabular}{|c|r|}
\hline Etapas Fiscales & Suma de total procesos \\
\hline Querellable & 1367639 \\
\hline Terminación anticipada & 5639 \\
\hline Indagación & 4762464 \\
\hline Etapa de investigación preliminar & 26426 \\
\hline Investigación & 69454 \\
\hline Etapa de instrucción & 4591 \\
\hline Juicio & 252482 \\
\hline Ejecución de penas & 310453 \\
\hline (En blanco) & 22 \\
\hline Total & 6799170 \\
\hline
\end{tabular}

Fuente: elaboración propia con base en datos de la FGN (2019-2020).

Ahora bien, con respecto a la tabla, esta muestra las diferentes etapas en las que se encuentran los procesos en curso, en relación con los datos reportados por la FGN, y la investigación que lleva a la escritura del presente artículo, llama la atención, con una elevada cifra la etapa de indagación, lo que hace inferir que este 70 \% no cumple con el acervo probatorio suficiente para llevar las causas ante el juez de control de garantías, en audiencia de formulación de imputación, sin lograrse la tipificación de la conducta punible por parte de la Fiscalía, dejando en riesgo de prescripción y caducidad los hechos denunciados, aunado a una 
ausencia de investigadores, un déficit en talento humano para apoyar las labores investigativas coordinadas por la FGN.

Si bien el legislador ha congregado esfuerzos para suplir este déficit, con la implementación del procedimiento abreviado, contemplado en la Ley 1826 de 2017, posibilitando a las víctimas apoyarse en un acusador privado, esto solo es procedente en asuntos querellables, y para quienes cuenten con los recursos pecuniarios para acceder a este servicio, perdiendo de vista el talento humano necesario para las denuncias.

También se puede evidenciar que, del total de los casos, el $20.11 \%$ son querellables, lo que implica que son causas que pueden pasar a terminación anticipada, las cuales en la tabla registran en un $0.08 \%$, entre conciliaciones y desistimiento de la víctima, actualmente.

Tabla 3. Procesos en la rama penal

\begin{tabular}{|c|r|r|}
\hline Juzgados & Ingresos efectivos & \multicolumn{1}{|c|}{ Egresos efectivos } \\
\hline Municipal garantías & 307369 & 284095 \\
\hline Municipal & 199965 & 178360 \\
\hline Circuito & 145470 & 112780 \\
\hline Especializado & 17328 & 16456 \\
\hline Total & 670132 & 591691 \\
\hline Total procesos & 435585 & 384599 \\
\hline
\end{tabular}

Fuente: elaboración propia con base en datos de la Rama Judicial (2019).

Por último, es importante tener en cuenta que de las 6 799-170 conductas punibles cometidas y que el $80 \%$ de ellos son recibidos ante un juez para su determinación y dar respuesta al proceso, existe una gran congestión, debido a la gran cantidad de procesos que ingresan, de los cuales el $12 \%$ quedaron sin respuesta durante el 2019. Un gran factor que influye a esta congestión, y el cual es pertinente mencionar, es que los jueces además de los procesos también deben dar respuesta a las acciones constitucionales 
que puedan llegar a presentarse. Por otro lado, también influyen los riesgos antes mencionados, ya que, si el fiscal tipifica una conducta punible que no se logra demostrar, mediante una teoría del caso y tiene ausencia de material probatorio, esto puede tener repercusiones en el devenir procesal, mediante una preclusión o absolución, que no siempre está ligado a la inocencia del indagado, incurriendo en impunidad.

Dicho esto, queda claro que la FGN es la única entidad con el deber de hacer una adecuación de la conductas típicas; sin embargo, es común encontrar que se den imprecisiones en la adecuación, lo que genera una afectación de la garantía constitucional del debido proceso, además de esto el proceso se puede encontrar entre las causales de archivo, lo que a su vez genera demora en el procedimiento, en razón a que surge un trámite adicional para recaudar nuevos elementos materiales probatorios o lo que en su defecto sea necesario para el desarchivo del proceso. Esto puede traer afectaciones, incluso más graves, pues al ser la FGN la única entidad encargada de adecuar la conducta es ella quien debe solicitar al juez que se reformule esta, por lo cual si esta inconsistencia pasa desapercibida por las partes se podría condenar a una persona más tiempo del que legalmente debía tener o, por otro lado, la disminución en la pena (Guarín y Arango, 2018).

Estas son las razones que llevan a abarcar la implementación de la IA en la fase de indagación que adelanta la FGN, con apoyo de la policía judicial, como un asistente con alcances decisorios que, una vez alimentado de la estadística reportada por la entidad, permita la identificación de la conducta punible con base en los hechos relatados por la víctima o quien realice la denuncia, disminuyendo el margen de error, y los términos procesales en cumplimiento de acceso efectivo a la administración de justicia, tutela judicial efectiva, principios de eficacia y eficiencia de la 
administración de justicia, contribuyendo a la descongestión judicial y ayudando en la identificación del acervo probatorio mínimo a recaudar para una exitosa presentación de la teoría del caso ante la autoridad judicial competente (Saidiza y Carvajal, 2016).

\section{La informática jurídica y la ubicación de la inteligencia artificial en el derecho}

En un ámbito tan amplio como lo es la tecnología, el ser humano ha realizado grandes esfuerzos para encontrar herramientas por medio de las cuales exista mayor facilidad de realizar diferentes labores, desde las más sencillas hasta las que tienen un alto nivel de complejidad (Patarroyo y Benavides, 2014). Es por ello que se han implementado sistemas informáticos capaces de agilizar actividades de diferente índole, dentro de las cuales se encuentran labores propias del área del derecho, tema de interés en el presente artículo.

Para dar un contexto de lo que es la informática, en general, es importante mencionar que esta noción agrupa diferentes procesos por medio de los cuales determinada información se controla y ordena. A lo largo del tiempo, el término ha sido relacionado con aparatos electrónicos, específicamente computadores, sin tener en cuenta que desde antes que estos aparecieran se podía apreciar la informática en un sentido más amplio; la escritura, los ficheros y expedientes son solo algunos ejemplos de cómo ha sido prescindible desde hace tiempo la organización de la información (Guibourg, 1998).

Para abarcar el vínculo que guarda el campo informático con el jurídico, es importante señalar que dicha relación recibe el nombre de informática jurídica, la cual consiste en la implementación de herramientas tecnológicas con las cuales se facilita la ejecución de procesos jurídicos

Via Inveniendi Et Iudicandi

e-ISSN: 1909-0528 | DOI: https://doi.org/10.15332/19090528

Vol. 16 N.0 2 | julio-diciembre del 2021 
(García, s. f.). En otras palabras, esto responde a aquellos sistemas informáticos que agilizan labores propias del campo del derecho.

Para ahondar en el concepto de la informática jurídica, resulta importante hacer mención de que esta, pretende no solo controlar y ordenar datos jurídicos, sino también automatizar diferentes actividades que realizan los operadores judiciales. Para comprender esto, de una manera integral, el concepto puede dividirse de la siguiente manera: tratamiento racional, tratamiento automático y contenido jurídico (Salazar, s. f.).

El tratamiento racional consiste en llevar a cabo procesos que son fundamentales para hacer un correcto traspaso de la información, desde su origen (sentencias, expedientes, actas, entre otros) hasta la base de datos o formato a la que se pretende hacer llegar. Los procesos en mención tienen que ver con el análisis y agrupación de la información para que de dicha manera exista una debida organización de los contenidos jurídicos, es por ello por lo que sin este proceso no sería posible hablar de informática y mucho menos de informática jurídica.

El segundo componente, correspondiente al tratamiento automático, supone la aparición de computadoras, mediante las cuales la información se procesa gracias a programas y algoritmos, herramientas fundamentales en la automatización que se busca. Finalizando con estas agrupaciones, se encuentra el contenido jurídico, sin el cual la noción perdería el sentido, pues su enfoque está dirigido al procesamiento de datos meramente judiciales (Salazar, s. f.).

Una vez establecido lo anterior, es importante abordar algunas de las características que el concepto de informática jurídica envuelve, vale la pena señalar que estas guardan relación directa con los componentes ya mencionados. Entre ellas se encuentra, en primer lugar, el estudio de las herramientas implementadas para la automatización, es decir, aquellas 
mediante las cuales se pretende procesar la información jurídica, lo que lleva a su vez a la siguiente característica, asegurar que los datos recibieron el respectivo tratamiento racional ya mencionado (Huertas, Leyva, Lugo, Perdomo y Silvero, 2016).

Por otro lado, se encuentra la participación de operadores judiciales, quiénes son fundamentales para el análisis de la información que se quiere ordenar, lo que lleva, a su vez, a la verificación de las computadoras, en el sentido de identificar si procesan correctamente los datos. Dicho esto, se puede decir que en la metodología de la informática jurídica existen dos fases, una en la cual se estudia el contenido y otra meramente técnica (Salazar, s. f.).

En la fase en la cual el contenido es estudiado, se realiza una labor manual, en la que la información pasa por un proceso de análisis integral, allí se busca también que los sistemas informáticos contribuyan a la facilitación de la búsqueda, es por ello que, mediante técnicas como las "palabras clave" o los "descriptores", se logra encontrar determinado contenido jurídico en una mayor brevedad, pues basta con una o varias palabras que guarden relación con el tema central para que este sea hallado (Salazar, s. f.).

Estudiado el concepto a grandes rasgos, es importante mencionar que este se divide en tres categorías, en el que se desarrolla la noción en general: informática jurídica documental, de gestión y decisoria. La primera pretende facilitar el almacenamiento de documentos de contenido jurídico, en tanto que recuperar información de esta índole puede ser una tarea que toma tiempo, pues cada vez aumenta la cantidad de datos frente a diferentes temas judiciales, es por ello, que resulta necesario un sistema que clasifique dicha información, facilitando así la tarea de búsqueda (Peña, 2014. 
Por su parte, la informática jurídica de gestión trata de un sistema tecnológico que, como su nombre lo indica, ayuda a gestionar de manera integral un gran número de datos, los cuales a su vez pueden ser manipulados, mediante los "sistemas de gestión de base de datos" (García, s. f.). Es decir que por medio de esta técnica se busca agilizar la tarea de los operadores judiciales, ofreciendo programas y sistemas que vayan en pro de brindar una base de datos con la información que se requiera. La informática jurídica decisional, por su parte, trata de un sistema tecnológico, el cual está en la capacidad de tomar decisiones, esto mediante la ayuda de herramientas informáticas capaces de realizar un razonamiento jurídico (Peña, 2008). Esta técnica está directamente relacionada con la IA, tema que será abordado a continuación, pues integra también lo que se ha analizado a lo largo del presente escrito.

La IA resulta ser una de las secciones de la informática, que consiste en un sistema capaz de replicar actividades que un ser humano haría normalmente, esto, por medio de una máquina apta para recibir datos, traducirlos y realizar comparaciones, arrojando así resultados que se asemejan al pensamiento humano (Guibourg, 1998). Es por ello que en la informática jurídica hablar de IA supone un sistema informático capaz de resolver problemas jurídicos de una forma similar a como lo haría un conocedor del derecho.

El sistema informático, a su vez, cuenta con tres elementos básicos, por un lado, la estructura de los datos, por el otro, la predeterminación de las normas que deben tenerse en cuenta para emitir una decisión, y, por último, el "motor de inferencia" que ejecutará la resolución del problema.

En relación con ello, son cinco las fases por medio de las cuales el sistema es creado, el primero de ellos consiste en un título del sitio web, adaptación de la máquina a la información, un equipo de programación y

Via Inveniendi Et Iudicandi

e-ISSN: 1909-0528 | DOI: https://doi.org/10.15332/19090528

Vol. 16 N. 02 | julio-diciembre del 2021 
finalmente el establecimiento del grupo de expertos. (Martínez, 2012, p. 830)

En las diferentes fases se encuentran algunas disciplinas que coadyuvan en el proceso de la automatización inteligente. Una de ellas recibe el nombre de “ciencias de la computación”, la cual resulta ser un motor fundamental en el desarrollo de la automatización pretendida, pues por medio de ella se estudia el funcionamiento y diseño de los computadores, contribuyendo así a que sea posible replicar conocimiento en la máquina.

En el estudio del funcionamiento y diseño de computadores se encuentran diferentes temas, los cuales son fundamentales para la eficacia del proceso, algunos de ellos son algoritmos, lenguajes de programación, ingeniería de software, arquitectura y programación, gestión de información, entre otros. En este conjunto de tópicos se encuentra uno del cual se desprende una base imprescindible para la ingeniería de la información, el sistema inteligente (Banda, 2014).

Un sistema inteligente busca automatizar determinadas tareas, para ello debe existir un "aprendizaje" de la máquina, lo cual se logra por medio de la computación simbólica, que responde a sistemas lógicos basados en conocimiento, para que así el comportamiento de la máquina se asemeje al de un ser humano (Banda, 2014).

Dicha computación simbólica deriva a su vez de los "modelos de sistemas expertos”, los cuales buscan constituir la base de la información. De dicha noción surge también el llamado "modelo conexionista y redes neuronales”, en este se procura la resolución de problemas que no llevan consigo algoritmos, es decir, se busca que la solución que ofrezca la máquina parta de la información almacenada (experiencia), como sucede en un ser humano, y aprenda de la información que recolecta (Martínez, 2012). 
Podemos apreciar cómo la materialización de la IA se ve reflejada por medio de diferentes sistemas, específicamente, y en el caso de nuestro interés, el sistema jurídico. Resulta relevante mencionar que los problemas que el sistema jurídico pretende resolver pueden ser clasificados como (i) problemas convergentes tipo 1, aquellos cuya solución resulta concreta, (ii) problemas convergentes tipo 2, en los que no se logra determinar la solución, pero esta no resulta imposible, y (iii) problemas divergentes, en los que existen múltiples soluciones (Martínez, 2012).

Determinar el tipo de problema es importante para seguir una ruta de acción, es por ello que después de esto, se procede a utilizar los "sistemas expertos jurídicos", estos son sistemas computacionales, los cuales procuran llevar a cabo procesos cognitivos, similares a los que realizan los conocedores del derecho, para así dar solución al asunto jurídico que se pretende resolver.

$\mathrm{Al}$ tratarse de un sistema experto cuenta con elementos tales como una base de información establecida, el factor racional para la toma de decisiones y la comprensión de lo que el usuario requiere para ofrecer determinado contenido. Este último elemento requiere a su vez de una interacción clara, es decir, resulta necesario que la persona se relacione con el programa para que este pueda tener una "idea" de su requerimiento. "Los sistemas expertos jurídicos se clasifican a su vez en cuatro tipos, sistemas basados en reglas de producción, modelo positivista explícito subyacente, modelo constructivista y modelo de razonamiento legal basado en casos" (Martínez, 2012, p. 832).

En términos generales, estas clasificaciones responden a formas en las cuales puede ser dirigida la información jurídica y así mismo desarrollada, pues en cada una de ellas se muestran alternativas y enfoques diferentes, los cuales responden al problema jurídico que se esté abordando. 
Todo lo anterior supone un gran impacto a nivel social, pues resulta complejo concebir que un instrumento informático realice actividades propias de un conocedor del derecho. Sin embargo, la inclusión de la informática jurídica, y a su vez de la inteligencia artificial al campo jurídico, ha arrojado resultados positivos, mostrando mayor orden de datos y agilización de labores (Martínez, 2012).

\section{Estudio de derecho comparado en aplicación de la IA}

Debido al avance que tiene día a día la IA son cada vez más los países alrededor del mundo que se han visto en la necesidad de implementar medios tecnológicos que ayuden a un mejor desempeño, bien sea laboral o académico, ya que esto representa mayor celeridad y eficacia en las tareas que se ejerzan con ayuda de la inteligencia artificial.

Argentina es uno de los países latinoamericanos que ha implementado estas innovaciones al servicio de la justicia, en consecuencia, crearon a Prometea, un dispositivo que funciona mediante comando de voz (por medio de dispositivo móvil) o escritura mediante un chat-bot, el cual es capaz de generar un concepto jurídico íntegro. De esta manera, los usuarios pueden interactuar como si lo hicieran con un asesor real, Prometea está en condiciones de realizar preguntas, las cuales la llevan a la búsqueda de leyes o decretos que sirvan para el caso en concreto y de esta manera formular un concepto jurídico, imprimirlo o enviarlo por correo electrónico para que este pase a revisión y sea corregido de ser necesario. Además de ello Prometea está programada para ayudar a la Fiscalía con sus expedientes, con solo ingresar el número de este, se arrojará una copia del expediente y así mismo un informe si es que están vencidos los plazos o hace falta algún requerimiento formal (Corvalán, 2018). Este avance es algo muy similar a lo que está sucediendo actualmente en Colombia con el 
robot Siarelis, el cual cuenta con un sistema de IA, programado para asesorar litigios societarios, siendo la Superintendencia de Sociedades de Colombia, el primer juzgado de América Latina que cuenta con un robot como asistente, algo que hace unas cuantas décadas habría sonado irracional.

Al igual que Prometea, Siarelis realiza una serie de preguntas al juez con base en los hechos y con esta información ofrece su opinión, además de sugerir, si acceder o no a las pretensiones y realiza una búsqueda de jurisprudencia de casos similares, el robot fue creado con el fin de ahorrar horas extensas de trabajo y así mismo darle celeridad a la resolución de demandas (Supersociedades, 2018). Existe aún una controversia frente a estas nuevas tecnologías, pues muchos piensan que un robot jamás va a poder reemplazar las labores de un humano, mucho menos pensar que pueda reemplazar la labor de un juez o un abogado. Sin embargo, como se puede observar, Latinoamérica le está apostando al desarrollo, un total acierto, debido a la manera tan acelerada en que crece la tecnología, no como reemplazos, pero sí como auxiliares, las nuevas tecnologías pueden aportar beneficiosamente a que haya un mayor acceso a la justicia de manera más rápida, eficiente y eficaz.

Canadá es uno de los países que cree completamente en la IA y el beneficio que esta trae al desarrollo de una nación, es por ello por lo que ha construido centros de investigación robótica, con el fin de experimentar y llegar a un conocimiento más profundo. En el 2016, Canadá concurrió junto con Estados Unidos y México para la renegociación del Tratado de América del Norte, negociación que llegó a su fin el 30 de septiembre del 2018, conocido como Acuerdo entre Estados Unidos, México y Canadá (TMEC), denominado por el presidente de Estados Unidos en ese momento, Donald Trump, "el acuerdo más grande en la historia”; sin embargo 
surgieron ciertas dudas frente a buscar modernizar el mecanismo de resolución de conflictos en materia de derechos antidumping, debido a que este mecanismo es considerado sumamente lento, causal que perjudica a las empresas que quieren solucionar sus conflictos con premura.

Existen actualmente herramientas que pueden ayudar y facilitar estos procedimientos (Nava, 2019). En concordancia con lo anterior, en la Universidad de Montreal de Canadá se desarrolló un laboratorio de ciberjusticia, con el cual pretenden tener un mayor acceso a la justicia, el principal proyecto fue nombrado: "Repensando la ley procesal: hacia la ciberjusticia", de allí se desprendieron varios subproyectos, uno de los cuales tiene el nombre de "Aplicación de semántica intertextual a la ciberjusticia" (CEJA, 2019) este nuevo concepto (ciberjusticia) es solo un avistamiento a la evolución tecnológica, y el interés a nivel mundial que tiene el modernizar la justicia o el acceso a ella mediante el uso de nuevas tecnologías.

Desde hace algunos años, se puede observar cómo ha cobrado importancia el uso de instrumentos tecnológicos y el acceso ilimitado a internet, el cual ha tenido un impacto al desarrollo en un aspecto jurídico, pues por medio de este ya podemos obtener de manera sencilla leyes, decretos, sentencias, algo que anteriormente era de limitado acceso. El concepto ciberjusticia se entiende como aquel que fusiona o integra las tecnologías de la información y de la comunicación en aquellos procesos que requieren la resolución de un conflicto, ya sea de forma judicial o extrajudicial (Duaso, 2019).

Por otra parte, en Australia están utilizando sistemas expertos jurídicos, en la Universidad de Melbourne fue creado el sistema experto basado en reglas y redes neuronales split-up, entiéndase por redes neuronales

Via Inveniendi Et Iudicandi

e-ISSN: 1909-0528 | DOI: https://doi.org/10.15332/19090528

Vol. 16 N.0 2 | julio-diciembre del 2021 
artificiales todas aquellas que están compuestas por procesadores que guardan información sobre determinados temas, que hacen fácil tener una interacción similar al comportamiento humano y esto es posible por medio de la recolección de información, mientras más información se ingrese a la red, más similitud con el comportamiento humano tendrá (Flores y Fernández, 2008).

De esta forma, la Universidad de Melbourne, especializada en temas de familia, implementó este sistema, el cual está dirigido a ayudar en la solución de conflictos de esta índole. El sistema está compuesto por un método que permite clasificar cada caso iniciando por la edad de los cónyuges, salud y posibilidades de empleo, solicita un recuento detallado de estudios realizados, así como de sus gastos primarios, si tienen enfermedades y los costos derivados, además de ello, su salario, la cantidad y en qué fecha lo recibe, todo esto en conjunto para determinar el porcentaje que les corresponde a los cónyuges, por ejemplo, para la manutención de sus hijos. Para que esto fuera posible, se requirió reunir y clasificar por temas cada sentencia para de ella poder extraer un común denominador y que de esta forma el sistema pudiera identificar un caso en concreto y realizar preguntas específicas para cada caso, y de estas ofrecer una predicción, la cual es útil para mediadores o abogados (Martínez, 2012).

Además de Australia, México también incursionó con Expertius, un sistema experto basado en el modelo constructivista y redes neuronales, el cual sirve como auxiliar en la toma de decisiones judiciales en los juicios de alimentos, fue llevado a cabo por el Departamento de Inteligencia Artificial Aplicada del Instituto de Investigaciones Jurídicas de la UNAM, también fue desarrollado un software ideado para ayudar a los jueces de

Via Inveniendi Et Iudicandi

e-ISSN: 1909-0528 | DOI: https://doi.org/10.15332/19090528

Vol. 16 N.o 2 | julio-diciembre del 2021 
familia a emitir sentencias en los juicios de divorcio, patria potestad y alimentos (Martínez, 2012).

España, para el 2018 comenzó con el desarrollo de varios sistemas entre los que se destacan Jurimetría, Tyrant Analitics y Vlex Analitics, los cuales servirían de apoyo para abogados jueces y funcionarios, la jurimetría fue diseñada con el fin de definir estrategias procesales idóneas frente a diferentes casos en discusión. Este sistema se alimenta de sentencias, autos y leyes actualizadas tomadas del Consejo General del Poder Judicial, Tyrant Analitics está direccionado hacia la modernización de la jurisprudencia, el programa recopila datos con el fin de plasmarlos en una serie de gráficos, los cuales son de carácter interactivo y generan facilidad a la hora de buscar jurisprudencia frente a un caso en concreto. Vlex Analitics, por otro lado, se creó para generar cálculos por medio de algoritmos de inteligencia artificial, este sistema puede realizar una predicción de pensiones compensatorias y el tiempo de la pena según el tipo penal también procesa sentencias de que extrae la información relevante que le puede ser de utilidad al abogado para preparar su litigio o al juez para emitir un fallo (Ayuda, 2019).

Se puede observar como todos los países mencionados tienen algo en común, su interés por incursionar, aprender, explorar y explotar todo lo referente a la IA, países que no ven esta herramienta como un enemigo para el ámbito jurídico, todos aciertan en que es un aliado para llegar a tener mayor acceso a la justicia y que esta sea rápida eficaz y eficiente. Algunos están más de lado de la robótica, otros de los sistemas jurídicos expertos, los cuales son los más cercanos para la aplicación de la IA en el derecho; sin embargo, no importa qué tipo de IA desarrollen, todo va direccionado hacia el mismo fin, la modernización, la inclusión de la ciberjusticia, el acceso en su totalidad a la justicia y la celeridad de esta. 
Por lo anterior, es imprescindible que en una era informática se instauren nuevos mecanismos para la ayuda de la justicia, de manera que se progrese al tener conocimiento de las dificultades con un estudio más detallado de la materia y teniendo como antecedente la inserción de esta con sus dificultades y fortalezas en los países europeos y americanos. Un ejemplo de ellos son los tribunales de 21 jurisdicciones en Estados Unidos, con ayuda de la Fundación Arnold han invertido gran cantidad de dinero para que la IA sea implementada en los procesos, esto se da para la valoración de las probabilidades de reincidencia de un procesado y también para mirar el porcentaje de posibles evasiones de la justicia antes de su juicio final (Završnik, 2020).

En Colombia, desde 2019, la Corte Constitucional, en labor conjunta con la Universidad del Rosario, el Laboratorio de Innovación e Inteligencia Artificial de la Universidad de Buenos Aires y el Ministerio Público Fiscal de la ciudad Autónoma de Buenos Aires suscribieron un memorial para el desarrollo de una prueba experimental, esta se afincó al proyecto denominado Prometea, el cual le da un respiro a la jurisdicción constitucional frente a tutelas de salud (C.C., Informe de gestión 20182019). Por otro lado, la Superintendencia de Sociedades cuenta con Siarelis, la cual presta asesoría a la Delegatura para los Procedimientos Mercantiles cuando se pretende decidir sobre casos societarios (Supersociedades, 2018).

Por otra parte, es importante mencionar a Watson, la IA que se implementa en la FGN, la cual empezó a funcionar a partir del 2018, y es un software que trabaja con big data; gracias a este se asocian casos del indiciado y permite el desarrollo de una investigación de contexto (FGN, 2018), es pertinente resaltar la funcionalidad de Watson, pues es un sistema de IA que demuestra que la justicia como mecanismo de control 
social ha comenzado a introducir nuevas herramientas tecnológicas, como lo es la IA en el derecho penal.

El software de Watson tiene acceso a la base de datos del SPOA y permite determinar si existen denuncias contra el mismo individuo, esto quiere decir que gracias a este sistema operativo se podrán reabrir o impulsar investigaciones. Watson analiza elementos similares que puedan contribuir con la investigación "modus operandi, características físicas, tipos de armas y vehículos, entre otros" (FGN, 2018).

De lo anterior, se alcanza a dilucidar que, en países extranjeros, así como en el nuestro, se ha optado por apostarle a la IA y resulta una herramienta muy útil para la solución de problemáticas jurídicamente relevantes; prestando una mayor eficiencia frente a la productividad y desarrollo de tareas que se recubren de gran complejidad. En el derecho esta es una herramienta que busca agilidad en el aparato jurisdiccional, generando una descarga laboral de actividades que resultarían mecánicas para la administración de justicia, esto hace que el operador se limite a centrarse en casos que requieren de su especial análisis y atención, sin dejar de contar con el apoyo de la herramienta informática que les permite tener la información organizada y precisa del caso en concreto.

\section{Recomendaciones para la implementación de un software en el sistema penal}

En razón a lo anterior, el presente artículo planteó la pertinencia de implementar un sistema informático que coadyuve la función encomendada a la FGN, conforme a lo esbozado con antelación se trataría de un sistema de IA, el cual se ubicaría entre el grupo de informática jurídica decisoria supervisada. 
Lo anterior contemplaría la creación de un software que sea alimentado con las denuncias, querellas y peticiones especiales presentadas por la autoridad competente en los últimos diez años; que adicional a esto reúna condiciones de calidad y casos exitosos, información necesaria para que el sistema "aprenda" e identifique la clasificación que a la fecha han realizado los fiscales en cumplimiento de sus funciones, con un margen de error humano y aceptable para que, posteriormente, pueda analizar casos similares, siendo capaz de eliminar la información que menos le sirva, evitando la repetición y saturación de este.

Para esto se tiene como ventaja los datos abiertos con que cuentan las entidades públicas y que son de libre acceso y los archivos sometidos a la Ley de Archivo General de la Nación, pues muchas de estas declaraciones reposan en medios virtuales y en plataformas como ORFEO y como desventaja, los parámetros con los que hasta la fecha la entidad ha agrupado la información, puesto que deben ser relevantes para la identificación del delito.

Con esto se pretende un modelo machine learning, es decir, un aprendizaje automático en el que el algoritmo no tendría que definir nada (Gago, 2017), ni tendría que descubrir nuevos delitos, pues los que tienen conocimiento son los desarrolladores, que le dirían al programa que si cumple unos ítems hay un resultado en un primer momento. El resultado es una probabilidad, no es un sí o un no, es estadística y puede arrojar resultados, por medio de porcentajes, generando la posibilidad de que sea una u otra tipificación de la conducta punible.

En una segunda fase, el software estará en capacidad de recibir la declaración de manera verbal o física, con lectura de voz y lenguaje natural que le permita asimilar palabras a las que ya ha aprendido y discriminado, generando un reparto interno entre los fiscales por unidades de delitos y 
otorgando alternativas para la tipificación del delito, como sugerencias frente al material probatorio mínimo y necesario que se debe reunir para la elaboración de la formulación de imputación y sustentación de la teoría del caso.

Este sistema acortaría los términos que actualmente les toman a los funcionarios de la FGN, en la adecuación del tipo penal, con base en las pruebas recaudadas, y disminuiría los casos de preclusión y absolución, así como las prescripciones y caducidad de las acciones o recepción de querellas ya caducadas. Este programa podría ayudar a que, desde un inicio, los procesos no se encuentren viciados y puedan ser sujetos de algún tipo de nulidad, acortando los términos judiciales (Mendieta, Goyte y Oviedo, 2020).

Es importante señalar que la IA en derecho, indistintamente al área de aplicación, siempre debe ser auditada por los profesionales idóneos y especializados en la materia con cierta periodicidad, en un primer momento, porque las máquinas no cuentan con el raciocinio de un humano, que ejemplificado parte del sentido común, pues para nadie es un secreto que en empresas como Google han tenido que apagar software al no ser asistido, pues con el tiempo comienzan a generar su propio lenguaje de entendimiento, que es indescifrable para el ser humano, lo que pondría en riesgo los derechos humanos, no solo de la presunta víctima, sino del imputado. Estos casos también suceden en el evento en que no se audite el contenido que esté procesando y el software comience a analizar sus propias respuestas para dar respuestas futuras con base en sus mismos resultados anteriores.

Se sugiere que esta IA sea vinculada a la ya existente, esta es Watson, que permite continuar y optimizar el proceso con la recolección de material probatorio con diferentes entidades. Es preciso señalar que la inserción de 
la informática en el derecho es un proceso que es inminente, por tal razón se debe buscar la manera de adherirnos a todos estos cambios, esta compenetración del derecho en la era digital trae unos retos, pues el derecho al ser el fenómeno normativo que rige las relaciones de los hombres se ve en la obligación de cambiar de manera paulatina con la era digital.

\section{Conclusión}

Por imperativo constitucional se ha permitido que el ius puniendi esté en cabeza del Estado por medio de la FGN para así garantizar a toda persona, natural o jurídica, el acceso a la justicia de forma permanente, evitando dilaciones jurídicas, investigando toda conducta que asuma características delictuales.

Según los datos estadísticos, pudo analizarse la gran congestión en dicho ente acusador, específicamente, en fase de indagación; es decir, existe una gran lista de procesos que esperan la adecuación de la conducta punible. Ante el avance desmedido de indagaciones que reposan en los diferentes despachos judiciales y el poco talento humano que auxilia la actividad de policía judicial, se identifica la necesidad de implementar la IA para lograr la descongestión judicial, celeridad y eficacia en la FGN.

Lo anterior tras evidenciar que la IA ha compenetrado de manera directa en la forma como funciona el derecho, pues a lo largo del presente artículo se ejemplificó cómo los países se interesan en conocer más sobre esta y así mismo implementarla en sus sistemas normativos con el propósito de optimizar la labor humana, sin que esto represente la sustitución del hombre por la máquina.

La implementación de la inteligencia artificial en el aparato judicial por medio de un sistema jurídico decisional supone una contribución a la 
jurisdicción penal en cuanto a la agilización de sus funciones, sin ignorar que, durante el desarrollo de esta, debe haber cooperación de los funcionarios públicos para ayudar al sistema con la recolección de datos de manera lógica y organizada.

Es decir, que al implementar la IA en el derecho penal se encuentran grandes beneficios, como son: (I) la agilidad o eficiencia de la justicia, (II) la seguridad jurídica que se le otorga a la víctima en cada caso, ya que esta no estará viciada por subjetividades humanas al momento de la tipificación del delito y (III) el efectivo y riguroso análisis que se debe tener en cuenta con base en los fundamentos de derecho para la toma de decisiones en un proceso.

\section{Referencias}

Ayuda, F. G. (2019). ¿Inteligencia Artificial y Derecho? Sí, pero ¿̇cómo? Revista Democracia Digital e Governo Eletrônico, Florianópolis, 2(18), 36-57. https://core.ac.uk/download/pdf/289998412.pdf

Banda, H. (2014). Inteligencia artificial: principios y aplicaciones. Escuela Politécnica Nacional de Quito.

Centro de Estudios de la Justicia de las Américas [CEJA], 2019. Estudios comparados sobre reformas $\mathrm{Al}$ sistema de justicia civil Australia y Canadá vol. II. https://biblioteca.cejamericas.org/bitstream/handle/2015/5640/Estudioscompara dos_AustraliayCanad\%C3\%A1_vII.pdf?sequence=4\&isAllowed $=\mathrm{y}$

Colombia. Ley 906 de 2004, art. 68. Código de Procedimiento Penal. Septiembre 1 de 2004. D.O. núm. 45.658.

Cotino, L. (2019). Ética en el diseño para el desarrollo de una inteligencia artificial, robótica y Big data confiables y su utilidad desde el derecho. Revista Catalana de dret públic, (58), 29-48. DOI: http://dx.doi.org/10.2436/rcdp.i58.2019.3303 
Corvalán, J. G. (2018). Inteligencia artificial: retos, desafíos y oportunidades - Prometea: la primera inteligencia artificial de Latinoamérica al servicio de la Justicia. Revista de investigaciones constitucionales, 5(1). DOI:

https://doi.org/10.5380/rinc.v5i1.55334

Corte Constitucional de Colombia [C.C.], 2019. Informe de gestión 2018-2019, [Colom.]. https://www.corteconstitucional.gov.co/transparencia/Informe\%20GestionPresid encia2018-2019.pdf

Corte Constitucional de Colombia [C.C.], Sala plena, septiembre 30, 2003, M.P.: M. J. Cepeda Espinosa, Sentencia C-873 de 03, [Colom.].

Duaso, R. (2019). Ciberjusticia: tecnologías e internet para el acceso a la justicia. Biblioteca Jurídica Virtual del Instituto de Investigaciones Jurídicas de la UNAM.

Flores, R. y Fernández, J. M. (2008). Las redes neuronales artificiales, fundamentos teóricos y aplicaciones prácticas. Gesbiblo.

Fiscalía General de la Nación [FGN] (2018). Fiscalía entra en la era de la inteligencia artificial para combatir la impunidad (en línea).

Fiscalía General de la Nación [FGN] (s. f.). Manual de policía judicial versión n.$^{\circ} 2$.

Guarín, V. y Arango, C. (2018). Línea jurisprudencial del error en la calificación en el Nuevo Sistema Penal Acusatorio. Universidad Libre, Seccional Pereira.

Gago, R. (2017). Uso de algoritmos aplicados a bases de datos genéticos. Universitad Oberta de Catalunya.

Gonzáles, P. E. (2007). La policía judicial en el Sistema Penal Acusatorio. Doctrina y Ley.

Guibourg, R. A. (1998). Bases teóricas de la informática jurídica. Universidad de Buenos Aires.

García, J. D. (s. f.). Introducción a la informática jurídica. Institución Universitaria Politécnico Grancolombiano.

Hernández, A. (2009). Imputación fáctica y jurídica. Conferencia pronunciada en las XXVII Jornadas Internacionales de Derecho Penal, realizadas los días 24, 25 y 26 de agosto de 2005. Bogotá, Universidad Externado de Colombia, 87-107.

Via Inveniendi Et Iudicandi

e-ISSN: 1909-0528 | DOI: https://doi.org/10.15332/19090528

Vol. 16 N.o 2 | julio-diciembre del 2021 
Huertas, O., Leyva, M., Lugo, L., Perdomo, W. y Silvero, A. (2016). Entre la minimización y la expansión del derecho penal: la presencia de Beccaria en el debate contemporáneo. Revista IUSTA, (44), 41-59. https://doi.org/10.15332/s1900$\underline{0448.2016 .0044 .02}$

Peña, C. A. (2014). Informática jurídica y derecho informático. C\&T - Universidad de Palermo (Argentina).

Nava, W. (2019). Inteligencia artificial para la solución de controversias del acuerdo comercial de América del Norte. Revista Jurídica IURA, 4(1), 29-43.

http://journal.upao.edu.pe/Iura/article/view/1370

Montenegro, A. y Posada, C. (1994). Criminalidad en Colombia - Borrador de Economía. Banco de la Republica. 4. 15.

Marcoux, Y. (2015). Aplicación de la semántica intertextual a la ciberjusticia: muchas verificaciones de la realidad por el precio de una. Balisage: The Markup Conference 2015.

Martínez, G. C. (2012). La inteligencia artificial y su aplicación al campo del derecho. Alegatos, (82), 827-846.

Rama Judicial. (s. f.). Modelo de Gestión del Sistema Penal Acusatorio - Abc del Sistema. En Manual Básico Sistema Penal Acusatorio, [Colom.].

Mendieta Pineda, L. M., Goyte Pierre, M. y Oviedo, I. Y. (2020). El juez de ejecución de penas y las medidas de seguridad en Colombia: un análisis crítico sobre sus orígenes, consagración normativa y funciones. Via Inveniendi Et Iudicandi, 15(1), 91-118.

Patarroyo, S. y Benavides, P. (2014). Rupturas asignificantes: revisiones críticas en torno al derecho. Via Inveniendi Et Iudicandi, 9(1), 7-31. https://doi.org/10.15332/s1909-0528.2014.0001.01

Saavedra, C., Saavedra, C. A. y Montaño, J. J. (2018). Relación entre informática jurídica y el derecho informático herramientas básicas para los profesionales del derecho y de la informática. Revista Atlante: Cuadernos de Educación y Desarrollo.

Saidiza, H. y Carvajal, J. (2016). Crisis del Estado de derecho en Colombia: un análisis desde la perspectiva de la legislación penal. Revista IUSTA, 44(1), 17-39. https://doi.org/10.15332/s1900-0448.2016.0044.01

Via Inveniendi Et Iudicandi

e-ISSN: 1909-0528 | DOI: https://doi.org/10.15332/19090528

Vol. 16 N.0 2 | julio-diciembre del 2021 
Salazar, E. (s. f.). La informática jurídica: itinerario, Métodos y Perspectivas. http://servicio.bc.uc.edu.ve/derecho/revista/idc20/20-9.pdf

Sarmiento Cristancho, D., Medina Velandia, S. y Plazas Estepa, R. (2017). Sobre la responsabilidad y su relación con el daño y los perjuicios. Via Inveniendi Et Iudicandi, 12(2), 101-115. https://doi.org/10.15332/s1909-0528.2017.0002.05

Superintendencia de Sociedades. (2018). El primer juzgado de América Latina en contar con un robot asistente.

https://www.supersociedades.gov.co/Noticias/Paginas/2018/Supersociedades-elprimer-juzgado-de-Am\%C3\%Agrica-Latina-en-contar-con-un-robot-asistente.aspx

Tamayo, F. L. y Norza, E. (2018). Midiendo el crimen: cifras de criminalidad y operatividad policial en Colombia. Revista Criminalidad, 6(3), 49-71.

Villabella, C. M. (2020). Los métodos en la investigación jurídica. Algunas precisiones. Cuestiones constitucionales. En E. Cáceres Nieto (coord.), Pasos hacia una revolución en la enseñanza del derecho en el sistema romano-germánico (Tomo 4, pp. 161-177). Universidad Nacional Autónoma de México; Instituto de Investigaciones Jurídicas.

https://archivos.juridicas.unam.mx/www/bjv/libros/13/6226/22a.pdf

Završnik, A. (2020). Criminal Justice, Artificial Intelligence Systems, and Human Rights. ERA Forum, 2O(4), 567-583. https://www.doi.org/10.1007/s12027-020-00602-0 